ORIGINAL ARTICLE

\title{
Measuring plasma salicylate concentrations in all patients with drug overdose or altered consciousness: is it necessary?
}

\author{
D M Wood, P I Dargan, A L Jones
}

Emerg Med J 2005;22:401-403. doi: 10.1136/emj.2003.010298

Background: Salicylate self poisoning is potentially fatal. Plasma salicylate concentrations can be used to guide management when taken in the context of clinical features of toxicity and acid base status. Previous studies in the USA and Hong Kong have shown that routine measurement of plasma salicylate concentrations in all overdose patients is inappropriate, but there have been no previous studies in the UK.

See end of article for authors' affiliations

.....................

Correspondence to:

Dr D Wood, Specialist

Registrar in Clinical

Pharmacology,

Pharmacology and Clinical

Pharmacology,

Department of Basic

Medical Sciences, St.

George's Hospital Medical

School, Jenner Wing,

Cranmer Terrace, London

SW17 ORE, UK; dwood@

sghms.ac.uk

Accepted for publication

12 January 2004
Methods: A retrospective case note study from 1 February 2001 to 31 January 2002 was undertaken at the emergency department of St. Thomas' Hospital, London. Records were reviewed and information on demographic data, history, details of salicylate overdose, and documentation of clinical features of salicylate toxicity recorded.

Results: In total, 722 patient episodes were identified, of which 596 case notes were available and appropriate for inclusion in this study. Plasma salicylate concentrations (range 15-428 mg/l) were detectable in 50 patients (three notes not available), of whom 38 had given a positive history. The history of salicylate poisoning had a sensitivity of $81 \%$ (95\% confidence interval (CI) 67 to $91 \%$ ) and the predictive value of a negative history of salicylate ingestion in not detecting salicylate concentrations was $98 \% 195 \%$ $\mathrm{Cl} 97$ to $99 \%$ ). Insufficient information on clinical features of salicylate toxicity was recorded in 569 patients (including 35 patients who had a history of salicylate ingestion).

Conclusion: History of salicylate ingestion has a high sensitivity and negative predictive value with respect to the detection of plasma salicylate concentrations. However, current practice indicates that insufficient information is obtained from patients about the clinical features of toxicity. Routine measurement of plasma salicylate concentrations is not required unless there is (a) a positive history of ingestion of salicylates or (b) a reduced level of consciousness or other reason limiting the validity of the history obtained, together with clinical features consistent with salicylate poisoning.
A lthough salicylate poisoning is not very common in the UK, it still accounts for $5-7 \%$ of all poisoning admissions to hospital and 30-40 deaths per year. ${ }^{1}$ Following poisoning with salicylates, the clinical symptoms are usually indicative of toxicity. Poisoning requiring therapeutic intervention (plasma salicylate concentration usually greater than $400-500 \mathrm{mg} / \mathrm{l}$ ) is usually indicated by the presence of symptomatic tinnitus and other symptoms or signs such as vomiting, tachypnoea, and sweating. ${ }^{2-4}$ Plasma salicylate concentrations are measured in a significant number of patients even when there is no clinical evidence of toxicity. At the time of this study, in the emergency department (ED) of St. Thomas' Hospital, London, all adult patients were screened for both paracetamol and salicylate poisoning following presentation with any suspected drug overdose. To assess the effectiveness and appropriateness of this investigation, we performed a retrospective analysis of all patients who had salicylate concentrations measured.

\section{METHODS}

A retrospective analysis was performed of all adult patients (age $>16$ years) identified by the hospital results reporting computer system as attending the ED at St. Thomas' Hospital, London, UK between 1 February 2001 and 31 January 2002 inclusive and who had had a plasma salicylate concentration measured. The emergency records and, where appropriate, the hospital inpatient records for these patients were reviewed using a standardised survey questionnaire by one author (DMW). The following data were obtained from the records: (a) demographic data; $(b)$ history and details of salicylate overdose, including dose ingested and timing; and (c) presence of any clinical features suggestive of a salicylate overdose (tinnitus, vomiting, tachypnoea, sweating, flushing, acid base disturbance, tachycardia, renal impairment, raised anion gap). Further details on confirmed salicylate overdoses were recorded, including plasma salicylate concentrations, other biochemical test results, and any management given.

\section{RESULTS}

Between 1 February 2001 and 31 January 2002, approximately 100000 patients attended the ED at St. Thomas' Hospital. In total, 722 patients had salicylate concentrations measured over the study period. There were 126 case notes (17\%) missing (123 had undetectable salicylate concentrations), thus 596 patients were enrolled in our study population, $312(52.3 \%)$ male and $284(47.7 \%)$ female.

\section{History of salicylate poisoning}

A history of salicylate overdose was obtained in 48 (8.1\%) of patients who had salicylate concentrations measured. Of these patients, 38 had a detectable salicylate concentration (range $47-428 \mathrm{mg} / \mathrm{l}$ ) and the remaining 10 patients had an additional history of self poisoning with other drugs, toxins, or ethanol. Salicylate was detectable in nine of the patients who did not give a history of salicylate poisoning (range 15$298 \mathrm{mg} / \mathrm{l}$ ). History of salicylate poisoning had a sensitivity of $81 \%$ (95\% confidence interval (CI) 67 to $91 \%$ ) and a 


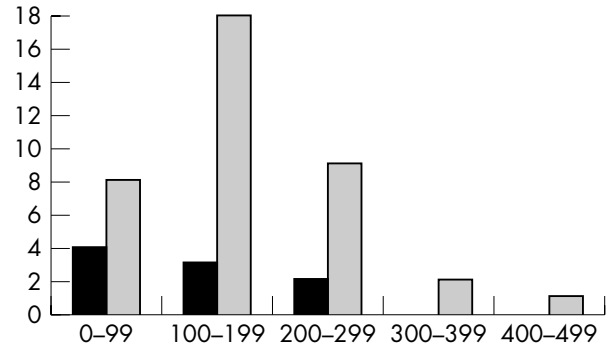

Figure 1 Number of patients with detectable salicylate concentrations (plasma salicylate concentration in blocks of $100 \mathrm{mg} / \mathrm{l}$ ) and positive or negative history of salicylate ingestion.

specificity of $98 \%$ (95\% CI 97 to $99 \%$ ) for the detection of salicylate in patients plasma. The predictive value of a negative history of salicylate ingestion that salicylate would not be detected was $98 \%$ (95\% CI 97 to 99\%). This means that screening only those patients with a history of salicylate ingestion would miss only $2 \%$ of laboratory confirmed cases of salicylate ingestion.

\section{Clinical features of salicylate poisoning}

Only 27 (4.5\%) patient episodes reviewed had sufficient information recorded in the admission cards concerning the presence or absence of symptoms and/or signs of salicylate poisoning. Of the 569 patients with insufficient information recorded on clinical features of salicylate poisoning, 35 had detectable salicylate concentrations, including the highest recorded concentration in this study of $428 \mathrm{mg} / \mathrm{l}$.

\section{Salicylate concentrations}

Of the 50 patients with detectable plasma salicylate concentrations, three admission records were not available. The highest detectable plasma salicylate concentration was $428 \mathrm{mg} / \mathrm{l}$, and no patient required intravenous sodium bicarbonate, urinary alkalinisation, or haemodialysis as part of the management of salicylate toxicity. Fig 1 shows the range of salicylate concentrations; in this study there was a greater frequency of positive history of salicylate ingestion in patients with higher plasma salicylate concentrations.

\section{DISCUSSION}

Incidences of alicylate poisoning have decreased in frequency in the UK, ${ }^{1}$ and consequently junior doctors in particular may not be as aware of the characteristic symptoms and signs. ${ }^{2}$ Initially, patients complain of sweating, nausea, and vomiting, and may have tinnitus, which tends to indicate a salicylate concentration $>400-500 \mathrm{mg} / \mathrm{l}$. Other signs of salicylate intoxication include sweating, flushing, bounding pulses secondary to peripheral vasodilatation, and initially a respiratory alkalosis. Signs of severe intoxication include a metabolic acidosis, renal failure, and central nervous system effects such as agitation, fitting, confusion, and coma. In all patients with suspected salicylate overdose, plasma salicylate concentrations should be measured at 2 hours in symptomatic patients or 4 hours in asymptomatic patients, ${ }^{5}$ but should be interpreted along with clinical state and any acid base disturbance. ${ }^{2}$ Salicylate forms concretions within the stomach, thereby delaying absorption, and plasma salicylate concentration measurements should be repeated until a peak has occurred. ${ }^{6}$

Several studies in the 1970s and 1980s suggested that routine toxicological screening in all overdose patients frequently identifies unsuspected drugs. ${ }^{7-9}$ Based on these, some authors argue that routine toxicological screening of all suspected overdose patients should be undertaken. However, the fact that a toxicological screen may pick up a drug that was not suspected is rarely of clinical importance, and the results of toxicological screens rarely result in changes in the management of patients, thus they are therefore not used routinely in clinical toxicology practice. ${ }^{10}$ The list of drugs for which a drug concentration can be useful as an adjunct to clinical parameters is small (paracetamol, theophylline, lithium, iron, salicylates, and anticonvulsants).. ${ }^{510}$

Several studies that have addressed the question of the clinical value of screening for salicylates in patients presenting with self poisoning. ${ }^{11-16}$ These have studied different population groups and have identified patients either by salicylate concentrations measured ${ }^{1214}{ }^{15}$ or review of consecutive patients with a diagnosis of suspected drug overdose, ${ }^{11} 1316$ and are summarised in table 1. The sensitivity (range 28-93\%) and negative predictive value (range 98$99 \%$ ) of history or clinical suspicion of salicylate ingestion in these studies are similar to those identified in our study. Two studies reported treatment interventions required in patients who were identified by laboratory analysis alone. ${ }^{11}{ }^{14}$ In both, maximum salicylate concentrations were below that requiring specific intervention for salicylate toxicity. Additionally, one was a follow up study at the same hospital following the group's initial results that showed that routine measurement of salicylate concentrations was not appropriate..$^{15}$ The follow up also showed similar results, suggesting that their initial findings had not altered clinical practice.

None of the above reported studies has commented on the physicians' accuracy and degree of reporting of clinical features of salicylate toxicity. The recording of clinical features suggestive of a salicylate overdose was poor in our study. In a retrospective notes study, it is difficult to ascertain whether the documented findings truly represent the clinical features on admission to the ED. However, it should be noted that these features were also not recorded in the initial assessment of patients who had admitted to salicylate ingestion. Documentation of clinical features of salicylate toxicity is important, as their presence is one of the main factors determining management of these patients. ${ }^{2}{ }^{4}$ Buckley

Table 1 Summary of previous studies that have investigated the clinical relevance of routine measurement of salicylate concentrations in patients presenting to emergency departments

\begin{tabular}{|c|c|c|c|c|}
\hline Study & Country & Method of identification of patients & $\begin{array}{l}\text { Sensitivity } \\
(\%)\end{array}$ & $\begin{array}{l}\text { Negative } \\
\text { predictive } \\
\text { value (\%) }\end{array}$ \\
\hline This study & UK & Positive salicylate concentrations & 81 & 98 \\
\hline Sporer et $a l^{22}$ & USA & Positive salicylate concentrations & 28 & $\mathrm{~N} / \mathrm{A}$ \\
\hline Brett et $a l^{11}$ & USA & Consecutive presenting overdose patients & 93 & 98 \\
\hline Mahoney et $a l^{77}$ & USA & Consecutive presenting overdose patients & 88 & 99 \\
\hline Chan et $a l^{15}$ & Hong Kong & Positive salicylate concentrations & 93 & 99 \\
\hline Chan et al ${ }^{16}$ & Hong Kong & Positive salicylate concentrations & 86 & 98 \\
\hline
\end{tabular}


et al have shown that the use of a pre-formatted admission chart for patients presenting with self poisoning increases the recording of clinical symptoms and signs of poisoning. ${ }^{17}$ We now use a standardised pre-formatted toxicology admission sheet for all patients presenting to St Thomas' Hospital with self poisoning, to increase the validity of data collected on our patients.

One of the potential limitations of our study is that there were no patients with serious salicylate poisoning during the study period. However, it supports the findings of previous studies in Hong Kong and the USA that routine measurement of plasma salicylate concentrations in all patients presenting with self poisoning is not of clinical value, particularly as one of the main factors used to guide treatment of these patients is the presence of significant clinical features of toxicity. ${ }^{2}{ }^{4}$ Thorough clinical assessment for the clinical features suggestive of salicylate intoxication (tinnitus, sweating, tachypnoea) would limit the number of salicylate concentrations measured in those patients who can give reliable histories. Patients with a reduced level of consciousness or significant intoxication with ethanol or other drugs, which could limit the validity of the history, or in whom the history is suspect should have salicylate concentrations measured.

Previous studies have shown that routine measurement of plasma salicylate concentrations following presentation with suspected self poisoning is inappropriate. This retrospective case note study shows that the patient's history has a high sensitivity and specificity for the detection of plasma salicylate. Additionally, insufficient information is obtained and recorded on clinical features suggestive of salicylate toxicity, including those patients with a documented history of salicylate ingestion. Routine measurement of plasma salicylate concentrations is not required unless there is $(a)$ a positive history of ingestion of salicylates or $(b)$ a reduced level of consciousness or other reason limiting the validity of history obtained, together with the presences of clinical features consistent with salicylate poisoning (such as coma, metabolic acidosis, respiratory alkalosis, tinnitus). These recommendations are in line with a joint position paper by the National Poisons Information Service and the Association of Clinical Biochemists. ${ }^{5}$

\section{Authors' affiliations}

D M Wood, Pharmacology and Clinical Pharmacology, St George's

Hospital Medical School, London, UK

P I Dargan, A L Jones, National Poisons Information Service (London), Guy's and St. Thomas' Hospital, London, UK

Competing interests: none declared

\section{REFERENCES}

1 Hawton K, Townsend E, Deeks J, et al. Effects of legislation restricting pack sizes of paracetamol and salicylate on self poisoning in the United Kingdom: before and after study. BMJ 2001;322:1203-7.

2 Dargan PI, Wallace $\mathrm{Cl}$, Jones AL. An evidence based flowchart to guide the management of acute salicylate (aspirin) overdose. Emerg Med J 2002; 19:206-9.

3 Krause DS, Wolf BA, Shaw LM. Acute aspirin overdose: mechanisms of toxicity. Ther Drug Monit 1992;14:441-51.

4 Temple AR. Acute and chronic effects of aspirin toxicity and their treatment. Arch Intern Med 1981;141:364-9.

5 Watson I. Laboratory analyses for poisoned patients: Joint position paper. Ann Clin Biochem, 2002;39, 328-39.

6 Jones AL, Dargan PI, eds. Salicylates (aspirin). In: Churchill's pocketbook of toxicology, 1st ed. London: Churchill Livingstone, 2001;2:80-4.

7 Hepler BR, Sutheimer CA, Sunshine I. The role of the toxicology laboratory in emergency medicine: II. Study of an integrated approach. J Toxicol Clin Toxicol, 1984-5, 22:50328.

8 Teitelbaum DT, Morgan J, Gray G. Nonconcordance between clinical impression and laboratory findings in clinical toxicology. Clin Toxicol 1977; 10:417-22.

9 Wright N. An assessment of the unreliability of the history given by selfpoisoned patients. Clin Toxicol 1980;16:381-4.

10 Dawson $\mathrm{AH}$, Whyte IM. Therapeutic drug monitoring in drug overdose. Br J Clin Pharmacol 1999;48:278-83.

11 Brett AS. Implications of discordance between clinical impression and toxicology analysis in drug overdose. Arch Intern Med 1988;148:437-41.

12 Sporer KA, Khayam-Bashi H. Acetaminophen and salicylate serum levels in patients with suicidal ingestion or altered mental status. Am J Emerg Med $1985 ; 14: 443-7$.

13 Baskin LB, Morgan DL. Drugs detected in patients suspected of acute intoxication. Tex Med 1997;93:50-8.

14 Chan TYK, Chan AYW, Ho CS, et al. The clinical value of screening for salicylates in acute poisoning. Vet Hum Toxicol 1995;37:37-8.

15 Chan TYK, Chan AYW, Ho CS. Use of a plasma salicylate assay service in a medical unit in Hong Kong: A follow-up study. Vet Hum Toxicol 1996;38:278-9.

16 Mahoney JD, Gross PL, Stern TA, et al. Quantitative serum toxic screening in the management of suspected drug overdose. Am J Emerg Med 1990;8:16-22.

17 Buckley NA, Whyte IM, Dawson AH, et al. Preformatted admission charts for poisoning admissions facilitate clinical assessment and research. Ann Emerg Med 1999;34:476-82. 